\title{
Collaborative Environments through Dialogues and PBL to Encourage the Self-directed Learning in Computational Sciences
}

\author{
Fernando Ramos-Quintana ${ }^{1}$, Josefina Sámano-Galindo ${ }^{2}$, and Víctor H. Zárate-Silva ${ }^{1}$ \\ ${ }^{1}$ Tecnológico de Monterrey, Campus Cuernavaca Autopista del Sol, KM 104 \\ Xochitepec, Morelos CP 62790. México. \\ ${ }^{2}$ PHD Student of Tecnológico de Monterrey and Teacher of Instituto Tecnológico de \\ Zacatepec, Calzada Tecnológico No. 27, Zacatepec Morelos, CP: 62780. México \\ \{fernando.ramos, A00334774, vzarate\}@itesm.mx, \\ jsamanog@gmail.com
}

\begin{abstract}
Self-directed learning has been one of the main objectives in the education domain. A learning model can drive a self-directed learning if an adequate educational environment is built. We propose an educational environment to encourage the self-directed learning, which is composed of a computer collaborative tool that uses dialogues and the concept of ill structured problems. The knowledge being learned is represented by a network of concepts built by the students through the exchanged messages. The network of concepts expressed the relation between the main concepts of the topic being learned. A coherent network is the tangible proof that the process of self-directed learning has been correctly achieved. Two topics of computer sciences are reported: Object Oriented Programming and Case Based Reasoning. The results have proven that along with the knowledge acquired, self-directed learning contributes directly to the development of skills for solving problems and attitudes of collaborative work.
\end{abstract}

Keywords: self-directed learning, collaborative learning, dialogues, ill structured problems.

\section{Introduction}

A concrete definition of self directed learning (SDL) was given by Gibbons: "In selfdirected learning (SDL), the individual takes the initiative and the responsibility for what occurs", [1]. Gibbons proposed some important elements to be taken into account in SDL, which are described as follows: student should control as much of the learning experience as possible; the development of skills; the self-challenge after having been challenged by teachers; self-management of their time, effort and the resources they need to conduct their work; self-motivation and assessment of their own efforts. Hiemstra [2] pointed out the following relevant aspects defining selfdirected learning: more responsibility of individual learners; self-direction is not necessary carried out in isolation from others; self-directed learners develop skills to transfer learning, in terms of both knowledge and study skill, from one situation to another; teachers in self-directed learning can dialogue with learners, evaluate outcomes and promote critical thinking. 
From the side of learners, Garrison affirmed that more responsibility and control are fundamental in self-directed learning [3]. Meanwhile, Ryan [4] mentioned that teachers in higher education need more responsibility for helping the students to develop self-directed learners. At the same time it was proposed problem-based learning as a potential framework to provide this assistance.

Teachers in higher education should assume more responsibility for helping students to develop as self-directed learners in their courses. In particular, problem-based learning is a potential educational framework within which it can be provided this assistance [4]. In the pediatric domain, Ozuah et al [5] is concerned with the effect of PBL on self-directed learning, concluding that pediatric residents exposed to PBL are engaged in significantly higher levels of self-directed learning than their counterparts. The use of problem-based learning (PBL) as an instructional methodology in undergraduate nursing curricula has been identified as one way to facilitate the development of nursing students' abilities to become self-directed in learning [6].

Ill Structured Problems (ISP's) are the vertebral column of Problem Based Learning. The ISP's are the key concepts used in the work presented in this paper to start the self-directed learning process. ISP's are similar as those found in real scenarios [7]. These types of problems represent important challenges to the students because their problem space is weakly defined. On the contrary, well structured problems (WSP) are those whose problem space has been well defined. In such a way that the bridge that joins an ISP with a WSP could be represented by the knowledge to be acquired [8]. An ill-structured problem can be a real problem able to incite the discovering of concepts and become the root for the construction of a network of concepts that represent the knowledge necessary to support a well understanding of a problem [9]. ISP's are commonly faced in everyday human experience; they have various solutions and have multiple solving processes depending on the solvers perception [10]. ISP's can be defined as problems that do not have known solutions. Experts in the domain do not agree regarding whether a particular solution is appropriate because it has various solutions and solution paths [11], [12], [13], [14].

Many of the problems we face in real life are ill-structured, including important social, political, economic, and scientific problems in the world [15], [14], [16].

Some authors cited above expressed the need of having additional elements to facilitate the self-directed learning. For instance, it has been proven that PBL facilitates the self-directed learning. Other additional elements can improve the facility for selfdirected learning. For instance, Hammond and Collins [17] recommended the construction of cooperative learning climate for self directed learning environments.

Collaborative learning has been defined by several authors as: a situation within which two or more people learn or attempt to learn something together [18] [19] [20]; in collaborative learning, the students work together to maximize their own learning [21]; collaborative group learning occurs when individuals interact through shared inquiry to construct their understanding of each other and their social worlds [22].

Several tools for working collaboratively have been proposed. The C-CHENE CSCL system was designed to facilitate computer-mediated interaction between human learners. The specific task studied requires students to construct qualitative models for energy storage, transfer and transformation using a specially designed graphical interface [23], [24]. C-CHENE uses two different communication interfaces to examine reflective interactions. A dialogue box allows the exchange of typewriting 
texts between learners in the first interface. Meanwhile the second one promotes an interaction using interface buttons for specific speech acts.

OSCAR is a framework based on the speech-acts theory that attempts to support students involved in collective activities and tutors in their perception of these activities by structuring textual communication through chats and forums. Woojin Paik et al [25] aim to develop intervention techniques to identify and remove obstacles of online learning groups. A secondary goal is to analyze the conversations of virtual group members using computational linguistics techniques. The final goal is to build an automatic system able to monitor the activities of the online learning group members to alert the instructors when the members encounter barriers.

Other authors are concerned with the study of dialogue patterns, which can help to build systems to assist the interactions student-student or student-teacher in the tasks of collaboration. Ravi and Kim [26] revealed the need of determining patterns of student interactions in online discussions for better information management and assistance. Pilkington [27] pointed out that formal and computational models of dialogue patterns extracted from the interaction of student-tutor and student-student interaction could help to bridge the gap between empirical investigation of interaction and the design of Intelligent Educational Systems (IESs) that interact with students.

In this work, the model of self-directed learning is supported by a collaborative computer tool called FreeStyler, developed by the Coollide group [28] [29]; ill structured problems; dialogues and draw actions whose purpose is to build a network of concepts that validate the knowledge acquired. The construction of a coherent network of concepts is made in several sessions. The network of concepts starts to be built as follows: given a concept represented by a circle node it will be transformed by a dynamic rectangle-node (transition) to get a new concept. The rest of the network is built following the same procedure. The exchanged messages of the dialogues contain collaborative actions to build the network. The network of concepts built in this work has certain similarity with the network of domain concepts built in Adaptive Hypermedia (AH) systems, where such network represents the user's knowledge of the subject [30] [31]. In AH systems, the concepts are related with each other thus forming a kind of semantic network which represents the structure of the subject domain. However, in the present work the links between concepts is given not only by static relations, but also by dynamic relations. For instance, a given concept is transformed into another one by applying actions, operations, mathematical methods, etc. As in Petri nets the transformations take place through special nodes representing transitions, as illustrated in Fig. 2.

The self-directed learning process proposed in this work can be carried out at two different levels of difficulty. The two levels are differentiated each other by the degree of participation of the instructor. At the first level, the instructor has provided the students with a set of basic concepts of a topic to be learned during a short presentation without treating in detail such concepts. Then, the students aim to reinforce or reaffirm themselves the knowledge weakly acquired previously. The reinforcement or reaffirmation of knowledge is dynamically carried out as they build a coherent network of concepts. The instructor validates the coherence of such network. As a result of this level, the students have learned the way of structuring the concepts of a topic and have developed skills of analysis and synthesis and abstraction. At the second level, the students are provided with an ill structured problem that contains constructors which 
guide the start of the self-learning process aiming to acquire new knowledge. As in the first level, the construction of a coherent network of concepts will validate that knowledge has been correctly acquired. The instructor in this case assumes the role of advisor, giving tips and suggestions, to build the network f concepts. He also validates the coherence of the network of concepts.

Both levels use the computer tool to exchange messages, so they build dialogues, and draw geometric figures to build the network of concepts. In this work, messages are classified as speech acts which change the state of the universe through the perlocutive effects due to the implicit actions involved in them as in the work of Allen and Perrault [32]. The topics to be learned are the following: Case Based Reasoning $(\mathrm{CBR})$ and Object Oriented Programming (OOP). It is described in this work the topic of OOP. CBR was at the level of reinforcement or reaffirmation of knowledge and OOP at the level of acquiring new knowledge.

This paper is organized as follows: section 1 introduced the context of the work, the relevant related works and a general description of the model proposed in this paper; in section 2 the process of self-directed learning proposed in this work is shown. In addition, it is illustrated how the ISP is provided to start the construction of the network of concepts and some details about the construction of the network; the analysis of results is treated in section 3. Although, the main focused of this analysis is on the performance of the self-directed process, short comments will be given about the analysis of dialogues and its importance in this research work; conclusions and future works are exposed in section 4; finally, important reference related with this work are provided in last section.

\section{The Process of Self-directed Learning}

Fig. 1 illustrates the process of self-directed learning proposed in this work. This scheme applies for the second level. As we can see, at the beginning of the process, the teacher provides the students with an ill structured problem (ISP). After carrying out an analysis (block I), the students make a self-identification of constructors and start the construction of the network.

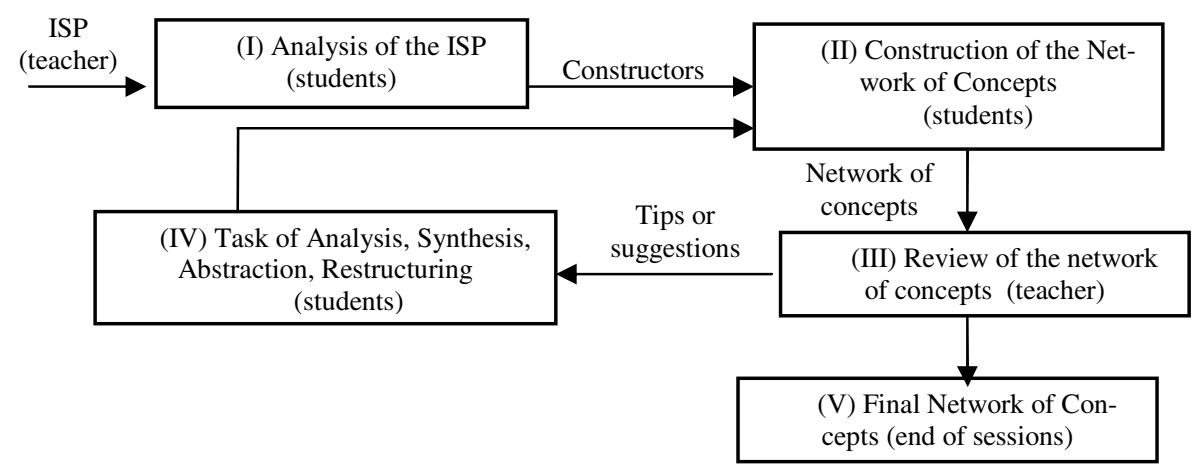

Fig. 1. The process of self-directed learning 
The output of block II is a network to be reviewed by the instructor in block III. He proposes tips or suggestions to update the network as output of block III. The students analyze, synthesize, make abstractions and reorganize the structure to update the network in block IV. The process is repeated until the end of the sessions or until the students get a coherent network of concepts.

\subsection{Building the Network of Concepts}

\subsubsection{Statement of the ISP}

For the learning of OOP topic an ISP should be exposed to the students. For instance, a statement of a real example could be the following: "the distribution of products of an industry can be modeled using the paradigm of Object Oriented Programming (OOP)". The constructors or openers extracted from the statement defined above are "distribution", "model" and "OOP". Most of the students choose to investigate the meaning of OOP. Teacher suggests investigating relevant concepts of OOP. Then, students build basic relations between two concepts, such as, the concept of "object" to be related with the concept of "class".

The students found several important concepts involved: object, classes, subclasses, simple and complex inheritance, methods, polymorphism and generalization, among the most important. During the collaborative sessions, the students built a network by linking concepts until a complete network is achieved (see Fig. 1, for the process of self-directed learning). Links represent desirable correct relations between concepts. The network is built dynamically as the messages are being exchanged, because messages contain actions. A guide or mentor assists the students in order to review the coherence of the network. The students could consult the mentor one time per session. Each session took $90 \mathrm{~min}$. The network was built during four sessions, which took one month.

\subsubsection{Construction of the Network of Concepts}

Fig. 2 shows the concept of "Objects" to which it has been applied the action of "Grouping" to obtain the concept of "Class". The corresponding dialogue to obtain the nodes related with Fig. 2 is shown below. The dialogues have been translated from Spanish to English in order to facilitate their reading in this paper. The predicates marked in italic represent drawing actions to build the network.

Express(A,J, "Hello") < Express (J,A, "What's up"?) < Question (J,A, "are you ready?") < Answer(A,J, "yes") < Proposition (A,J, Now, "we have to begin with class and object, ok?") < Accept(J,A, "Yes") < DrawNode(A,Objects) < DrawNode (A,Class) < DrawTran$\operatorname{sition}(A$, Grouping)

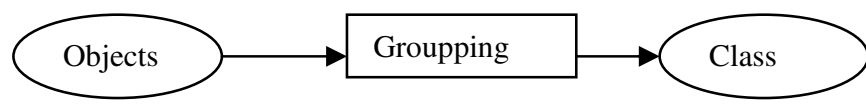

Fig. 2. The construction of the Class concept from the concept of Objects

The process to build the network is based on the one described in Fig. 1. The rest of the dialogue and the process of the construction of the whole network is not showed because lack of space. Fig. 3 shows an example of the whole network of concept obtained by one of the pair of students. The environment to build the network 


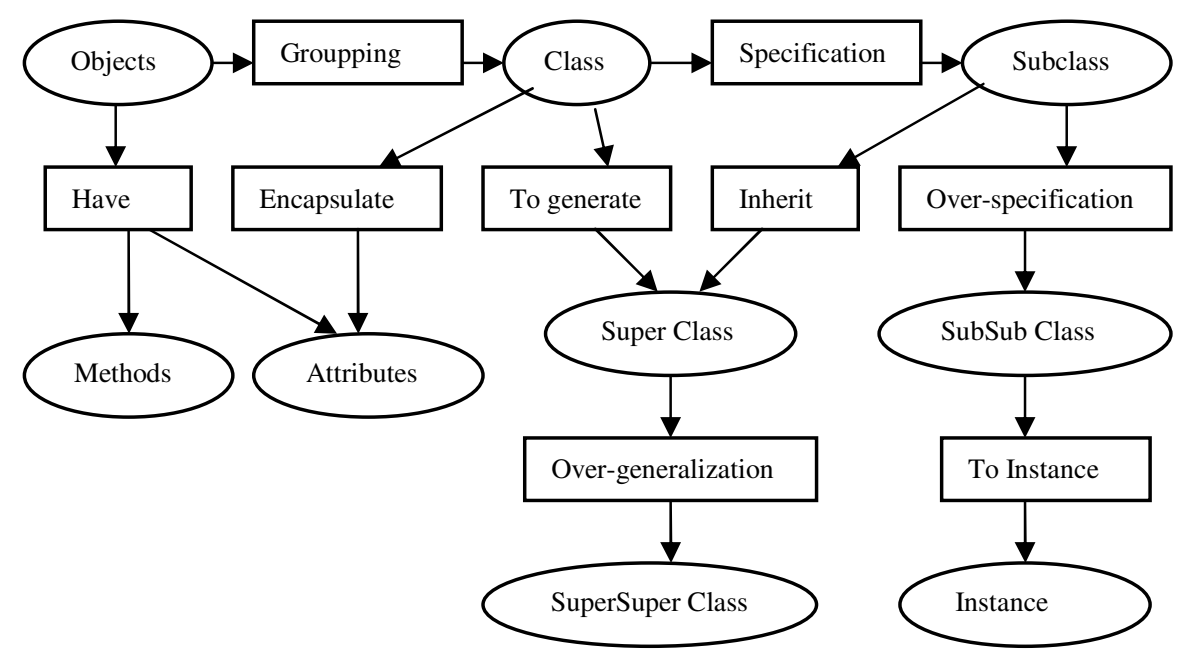

Fig. 3. An example of a whole network of concepts

includes a window to exchange message and a menu of geometric figures to draw the network.

\section{Analysis of Results}

24 pairs of students participated in "Case Based Reasoning" and 20 pairs in OOP. The task of reinforcing knowledge previously learned was focused on CBR. Meanwhile, the task of acquiring new knowledge was focused on OOP, which took four sessions during one month. Although, the main interest was to build a coherent network of concepts, we were also interested in measuring the development of skills to analyze, synthesize and build structures. These variables have been measured through the process of building the network of concepts based on a bottom-up approach. That is, at the bottom level the students should list the main concepts of the topic under study; then, at intermediate level, they should establish the main relationships between pairs of concepts, for instance, objects are grouping to form classes of objects; and finally at the top level they should assembly the whole network of concepts.

Then, it was measured the progress of the students to build correctly the network of concepts. For the analysis aspects, we were concerned about the capacity of students to establish the single and relational concepts to be used in the construction of the network. For the synthesis aspects, the students should build a synthesized but expressive network. For the construction of a structured network, it should reveal the construction of a coherent network, where the whole network should have a correct semantic meaning. We will show only the analysis of results related with OOP technology. In a good analysis, students should be able of extracting the following single concepts and establishing the correct relations between them.

At the bottom level of abstraction, the main concepts of OOP: Object, Attributes, Class, Subclass, Subsubclass, Instance, Instantiation, Grouping, Generalization, 
Over-generalization, Specification, Over-specification, Inheritance, Characterization, Metaclass, Superclass.

At intermediate level of abstraction, the main relationships between concepts: Object-Grouping-Class; Class-Specification-Subclass; Subclass-Generalization-Class; Class-Overgeneralization-Superclass; Attributes-Characterization-Object; SubclassOverspecification-Subsubsubclass; Subclass-instantiation-Instance; Class-Inheritancesubclass.

At the top level the network of concepts should be as that one shown in Fig. 3. In spite that the experience for reinforcing or reaffirming the knowledge of CBR was important to develop skills of analysis, synthesis and build structures, an average of $70 \%$ of the whole network of concepts was reached by the couples in OOP after the first two sessions. This is due to several reasons: the couples had different background knowledge in computer subjects; the autonomy attitudes were not developed at the same degree; the students had not the same level of capacities of analysis, synthesis and construction of structures; finally the mentor-guide assisted the students only two times as strategy to encourage the autonomy work of the students. The guide gave tips or suggestions but neither corrections nor solutions. At the end of the four sessions we have obtained the following results: 11 couples got a network $100 \%$ correct; 4 couples got one or two no relevant errors, which can be judged as $90 \%$ correct; 3 couples got three not relevant errors and 2 couples got four errors, two of them very important from the point of view of the coherence of the network. As an example, an important error is when sub-classes were not obtained from classes.

The same exercise of building a network of concepts was applied to 24 pair of students having a previous learning in OOP in a traditional fashion. The results shown that only 10 pairs of the students built a network $100 \%$ correct, requiring an important assistance of the guide. The rest of the students got an average of more than four mistakes. For instance, they were not able to extract the most important concepts. Consequently, the construction of relations was incomplete. This kind of errors resulted in an incoherent network of concepts. The skills of analysis and synthesis and construction of structures were weak in this group.

Based on the results obtained, we consider that the environment composed of the computer collaborative tool through dialogues and PBL, in particular ISP's, helped to encourage the self-directed learning. This kind of environments induces the development of skills such as analysis, synthesis and construction of structures.

An advantage of the FreeStyler tool is that it allows the students to communicate in a natural way. However, it is advisable to determine a set of rules to carry out the communication, otherwise, because there are not restrictions about the way of exchanging messages, students can loose the control without taking care of the main purpose of the conversation. The computer tool could have openers to build the network in order to help students to start the construction of it. These openers could be a list of single and relational concepts relevant to the topic under study.

This work is focused on self-directed learning process and the environment adequate for this purpose. However, we consider the importance of pointing out the work made to study the behavior of students through the dialogues or sequences of exchanged messages between students. The goal in this case was to discover patterns of dialogues capable of revealing us certain behavior of students as messages were being 
exchanged. Thus, if dialogue patterns exist then they could serve as guide to monitor and advice the students in case of deviations or obstacles as the network of concepts is being built. We have discovered patterns of dialogues related with phases of salutations satisfying the protocol of saying hello, at the beginning of the dialogue, and goodbye, at the end of the dialogue. At an intermediate phase the students start and finish, practically, the construction of the network. An overlapping between neighbor phases was characterized by an action of drawing indicating the start and the end of the network construction, respectively.

Some of the most important factors of distraction can occur in the initial and final phase, just where the students establish dialogues about the protocols of saying hello or good bye. The kind of distractions could be related with cultural factors.

Other patterns of sub-dialogues emphasized the illocutive force of a speech act within the sequence of the dialogue. The specialized sub-dialogues can be: ESD (Expressive Sub-Dialogue); QSD (Question Sub-Dialogue); PSD (Proposition SubDialogue); RSD (Request sub-dialogue).

\section{Conclusions}

To encourage the self-directed learning is needed a propitious environment. The environment proposed in this work was composed basically of a computer collaborative tool through which students exchanged messages to build a network of concepts. In addition, PBL helps, through Ill Structured Problems (ISP), to challenge and detonate the interest for investigating concepts around the topic being studied.

The idea of increasing the degree of difficulty to become self-learner starting with reaffirmation or reinforcing knowledge to the acquisition of new knowledge helps to develop and/or improve skills such as analysis, synthesis and construction of structures. These skills are necessary for improving the results in self-directed learning. We could verify that students can improve their skills to analyze, synthesize and build structures through the construction of a network of concepts. In addition, the proposed environment encourages students to improve attitudes for autonomy and team work. We have confirmed that the efficiency to build the network of concepts made by students that learned by the self-directed method was better than the efficiency got by students that learned under traditional methods.

A tool such as the one used in this work is adequate to work by pairs of students, more than two students could become the use of the tool very complicated. The students have used their own way of speaking. The culture and language are factors that should count certainly in the number of messages exchanged to achieve the task of building the network of concepts. The risk of distractions can occur if students are not concentrated in the objectives, which can increase the number of useless messages exchanged. Thus, one of the purposes of discovering dialogue patterns is to build an assistance computer system that can aid the students when they find obstacles or to end deadlocks.

We consider that the patterns are in a certain way generalized because they were independent on the pairs of students. However, we need more than two or three domain of studies, other than CBR or OOP, and the study under other cultural environments and languages to say that the patterns are generalized. 


\section{Reference}

1. Gibbons, M.: The Self-Directed Learning Handbook. Wiley, Chichester (2002)

2. Hiemstra, R.: Self-directed learning. In: T.H.T.N.P. (ed.) The International Encyclopedia of Education, 2nd edn., Pergamon Press, Oxford (1994)

3. Garrison, D.R.: Critical Thinking and Self-Directed Learning in Adult Education: An Analysis of Responsibility and Control Issues. Adult Education Quarterly 42(3), 136-148 (1992)

4. Ryan, G.: Student perceptions about self-directed learning in a professional course implementing problem-based learning. Studies in Higher Education 18(1), 53-63 (1993)

5. Ozuah, P.O., Curtis, J., Stein, R.E.K.: Impact of Problem-Based Learning on Residents' Self-directed Learning. Arch. Pediatr. Adolesc. Med. 155, 669-672 (2001)

6. Williams, B.: The Theoretical Links Between Problem-based Learning and Self-directed Learning for Continuing Professional Nursing Education. In: Teaching in Higher Education, pp. 85-98. Routledge (2001)

7. Jones, D.: What is PBL: Going Beyond Content, San Diego State University (1996)

8. Ramos, F., Espinosa, E.: A model based on Petri Nets for guiding the process of learning concepts needed to transform ill-structured problems into well-structured ones. In: PBL 2004 International Conference, Cancun (2004)

9. Ramos, F., Espinosa, E.: A self-learning environment based on the PBL approach: an application to the learning process in the field of robotics and manufacturing systems. The International Journal of Engineering Education 19(5) (2003)

10. Hong, N.S.: The relationship between well-structured and ill-structured problem solving in multimedia simulation, Pennsylvania State University (1998)

11. Jonassen, D.H.: Instructional Design Models for Well-Structured an Ill-Structured Problem-Solvin Learning Outcomes. ETR\&D 45(1), 65-94 (1997)

12. Reitman, W.R.: Cognition and thought, p. 312. Wiley, New York (1965)

13. Voss, J.F.: Problem solving and reasoning in ill-structured domains. In: C.A. (ed.) Analyzing everyday explanation: A casebook of methods, pp. 74-93. SAGE Publications, London (1988)

14. Voss, J.F., Means, M.L.: Toward a model of creativity based upon problem solving in the social sciences. In: Glover, R.R.R.J.A., Reynolds, C.R. (eds.) Handbook of creativity, pp. 399-410. Plenum, New York (1989)

15. Sinnott, J.D.: A model for solution of ill-structured problems: Implications for everyday and abstract problem solving. In: J.D.S. (ed.) Everyday problem solving: Theory and applications, pp. 72-99. Praeger, New York (1989)

16. Voss, J.F., Lawrence, J.A., Engle, R.A.: From representation to decision: An analysis of problem solving in international relations. In: Complex problem solving, Lawrence Erlbaum, Hilldale (1991)

17. Hammond, M., Collins, R.: Self-Directed Learning: Critical Practice. Nichols/GP Publishing, 11 Harts Lane, East Brunswick, NJ 08816. 250 (1991)

18. Dillenburg, P., et al. (eds.): The evolution of Research on Collaborative Learning. Learning in Humans and Machines. Spada \& Reimann (1996)

19. Dillenburg, P., Neville Bennet, E.D., Vosniaou, S., Mandl, H.: Collaborative Learning, Cognitive and Computacional Approaches. Advances in Learning and Instruction Series. Van Someren, Reimann, Boshuizen \& De Jong Elsevier Science Ltd, Oxford (1999)

20. Collazos, C.A., et al.: Evaluating Collaborative Learning Process, in Department of Computer Science, Universidad de Chile, Santiago, Chile (2001) 
21. Johnson, D.W., Johnson, F.P.: Joining Together, Group Theory And Group Skills. Allyn And Bacon (2000)

22. Ziegler, M.F., Paulus, T.M., Woodside, M.: Making Meaning Through Dialogue in a Blended Environment. Journal of Transformative Education (4), 302 (2006)

23. Lund, K., Baker, M.J., Baron, M.: Modelling Dialogue and Beliefs as a Basis for Generating Guidance in a CSCL Environment. In: Lesgold, A., Frasson, C., Gauthier, G. (eds.) ITS 1996. LNCS, vol. 1086, pp. 206-214. Springer, Heidelberg (1996)

24. Baker, M.J., Lund, K.: Promoting reflective interactions in a computer supported collaborative learning environment. Journal of Computer Assisted Learning (13), 175-193 (1997)

25. Paik, W., Lee, J.Y., McMahan, E.: Facilitating collaborative learning in virtual (and sometimes mobile) environment. In: Bussler, C.J., Hong, S.-k., Jun, W., Kaschek, R., Kinshuk, Krishnaswamy, S., Loke, S.W., Oberle, D., Richards, D., Sharma, A., Sure, Y., Thalheim, B. (eds.) WISE 2004 Workshops. LNCS, vol. 3307, pp. 161-166. Springer, Heidelberg (2004)

26. Ravi, S., Kim, J.: Profiling Student Interactions in Threaded Discussions with Speech Act Classifiers. In: Proceedings of AI in Education Conference (AIED 2007) (2007)

27. Pilkington, R.: Analyzing Educational Dialogue Interaction: Towards Models that Support Learning. International Journal of Artificial Intelligence in Education (12), 1-7 (2001)

28. Hoppe, U.: COLLIDE. Faculty of Engineering, University of Duisburg-Essen (2006), http: / / www. collide. info/background

29. Muehlenbrock, M., Tewissen, F., Hoppe, U.: A Framework System for Intelligent Support in Open Distributed Learning Environments. In: Proceedings of 8th World Conference on Artificial Intelligence in Education (AIED 1997), Kobe, Japan, August 20-22 (1997)

30. Brusilovsky, P.: Methods and techniques of adaptive hypermedia. User Modeling and User-Adapted Interaction Journal 6, 87-129 (1996)

31. Brusilovsky, P.: Adaptive Hypermedia. In: UMUAI: User Modeling and User-Adapted Interaction, Netherlands. pp. 87-110 (2004)

32. Allen, J.F., Perrault, C.R.: Plans, inference, and indirect speech acts. In: Annual Meeting of the ACL Proceedings of the 17th annual meeting on Association for computational Linguistics, Morristown, NJ, Association for Computational Linguistics, La Jolla, California (1979) 